Treating anxiety and depression in dementia

\title{
Meta-analysis of psychosocial interventions for people with dementia and anxiety or depression
}

Dr Deirdre Noone ${ }^{1}$, Dr Josh Stott ${ }^{1}$, Dr Elisa Aguirre ${ }^{1,2}$, Kelly Llanfear ${ }^{3}$ and Dr Aimee Spector $^{1}$

${ }^{1}$ Department of Clinical, Education and Health Psychology, University College London, ${ }^{2}$ North East London NHS Foundation Trust, Research and development Department, Goodmayes Hospital, Barley Lane, Ilford, IG3 8XJ. ${ }^{3}$ The Wolfson Neurorehabilitation Centre, St Georges University Hospital NHS Foundation Trust.

Corresponding Author: Deirdre Noone, Deirdre.noone.14@ucl.ac.uk,

Abstract word count: 227

Main text word count: 3743

Number of tables/figures: 6

Running Title: Treating anxiety and depression in dementia 


\begin{abstract}
Objectives: Assess the effectiveness of psychosocial interventions for depression and anxiety in people with dementia (PWD) or mild cognitive impairment (MCI). Method: OvidMedline, PsychInfo and Embase were searched for studies on the $5^{\text {th }}$ August 2017. The efficacy of the studies was estimated using meta-analyses. Results: Eight RCTs were included. No RCTs were identified for people with $\mathrm{MCl}$. Four RCTs found that psychosocial interventions (multicomponent intervention, Tai Chi, problem adaptation therapy and exercise/walking) were effective at reducing symptoms of depression in PWD who were depressed. One study (Tai Chi) found that these reductions were no longer evident at six-month follow-up. Another study, not included in the meta-analyses, found that pleasant events behaviour therapy and problem solving behaviour therapy improved depression symptoms and this effect remained significant at follow-up. Three RCTs found that psychosocial interventions (music therapy and cognitive behavioural therapy (CBT)) reduced symptoms of anxiety in PWD who were anxious. Evidence from two of these RCTs (music therapy and CBT) showed that these improvements were evident at three to six-month follow-up.
\end{abstract}

Conclusion: The identified psychosocial interventions are effective at reducing symptoms of depression or anxiety in PWD experiencing these symptoms. This review is limited by the quality of studies, small sample sizes and the heterogeneity of the interventions, therefore high quality studies with larger sample sizes are required to test the efficacy of specific interventions such as CBT.

Key words: dementia, mild cognitive impairment, psychosocial interventions, depression, anxiety. 


\section{Introduction}

An increase in life expectancy has resulted in an aging population, with the proportion of older adults predicted to double by 2050 (UN Department of Economic and Social Affairs, 2013). Therefore, interventions for age-related health conditions, such as dementia and mild cognitive impairment $(\mathrm{MCl})$ are becoming a focus of clinical practice and research (Kaplan \& Berkman, 2011). Depression and anxiety, common amongst PWD and MCI (Kane \& Terry, 2015; Lyketsos et al., 2002; Monastero, Mangialasche, Camarda, Ercolani, \& Camarda, 2009), are linked to poorer outcomes, such as reduced quality of life, worsened cognition, increased functional impairment, behavioural disturbance and mortality rates (Kales, Chen, Blow, Welsh, \& Mellow, 2005; McCurry, Gibbons, Logsdon, \& Teri, 2004; Rapp et al., 2011; Shin, Carter, Masterman, Fairbanks, \& Cummings, 2005; Starkstein, Jorge, Petracca, \& Robinson, 2007). As there is limited support for the effectiveness of psychotropic medication for depression and/or anxiety in PWD and MCI (Devanand et al., 2003; Leong, 2014; Moretti, Torre, Antonello, \& Pizzolato, 2006; Sepehry, Lee, Hsiung, Beattie, \& Jacova, 2012); depression and anxiety have been identified as specific targets for psychosocial interventions (Cooper, Sommerlad, Lyketsos, \& Livingston, 2015).

There have been several reviews and meta analyses in related areas (Olazarán et al., 2010; Orgeta, Qazi, Spector, \& Orrell, 2014; Regan \& Varanelli, 2013; Teri, McKenzie, \& LaFazia, 2005). In a systematic review of psychosocial interventions for depression in PWD, psychosocial interventions were found to improve depression in seven out of the eleven RCTs reviewed (Teri et al., 2005). A recent Cochrane review of psychosocial interventions for depression and anxiety in PWD and $\mathrm{MCI}$, found that 


\section{Treating anxiety and depression in dementia}

psychosocial interventions reduced symptoms of depression and anxiety. However, there were no available RCTs including MCl (Orgeta et al., 2014). The inclusion criteria in these reviews (Orgeta et al., 2014; Teri et al., 2005) did not stipulate that studies needed to recruit participants that met clinical criteria for depression or anxiety at baseline. This limits the scope of these reviews to provide guidance for evidence based clinical care, such as the NICE guidelines, because the results may underestimate the effectiveness of psychosocial interventions because of low baseline scores.

A systematic review of community-based psychological interventions that aimed to improve depression, anxiety, or adjustment in people with early dementia or $\mathrm{MCl}$. Psychological interventions were found (seven RCTs and eight pre-post studies) to have positive effects on depression scores (Regan \& Varanelli, 2013). Sub analysis was done on studies whose inclusion criteria stipulated that participants met criteria for depression at baseline (three RCTs and one pre-post study). Three studies showed that symptoms of depression improved for those that were experiencing depression at baseline. There was no sub analysis done on studies whose inclusion criteria stipulated that participants met criteria for anxiety at baseline.

The current review extends previous reviews by completing a systematic review, with meta-analyses, to compare the efficacy of psychosocial interventions that focus exclusively on PWD or those with MCI meeting clinical criteria for depression or anxiety at baseline.

\section{Methods}




\section{Treating anxiety and depression in dementia}

The PICOS framework (Petticrew \& Roberts, 2006) was used to select studies. Inclusion criteria

Population: Participants that: (a) met criteria for dementia according to the DSM-IV or International Classification of Diseases-10 (ICD-10), or (b) met criteria for MCI (Petersen et al., 2014). They also met criteria for either clinical depression or anxiety on a standardised outcome measure or a clinical interview on the baseline assessment, which was stipulated as an inclusion criterion in the study. Participants could be recruited from any setting.

Intervention: Psychosocial interventions that specifically targeted depression or anxiety symptoms. Psychosocial interventions were defined as interventions that focused on psychological or social factors (such as psychotherapy or an activity group), as opposed to a pharmacological intervention (Forsman, Schierenbeck, \& Wahlbeck, 2011; Frederiksen, Farver-Vestergaard, Skovgard, Ingerslev, \& Zachariae, 2015). Interventions could include caregivers or family members. Interventions for caregivers alone were not included. Interventions were included if they used a structured time-limited intervention between a participant and a facilitator, such as a weekly one-hour psychotherapy session that ran for eight weeks. The intervention could be delivered to individuals or groups. Comparator: The intervention could be compared to a control group; including treatment as usual (TAU), waitlist controls or a comparison intervention that could act as a control for the intervention under investigation.

Outcomes: Studies were included if they reported at least one standardised measure of either depression or anxiety.

Study design: RCTs that collected outcome measures at baseline and post treatment. There was no restriction in the sample size used in studies. 
Treating anxiety and depression in dementia

Publication characteristics: Published in peer-reviewed journals, in English at any time prior to the 4th August 2017.

\section{Exclusion Criteria}

- Quasi-experimental studies, case studies and qualitative research.

- Pharmacological interventions.

- Conference/poster presentations.

- If more than one article presents the same data, only one article will be presented in the review. The original article was used in these instances.

\section{Selection of Studies}

Relevant studies were identified using three search strategies.

(1) A systematic search was conducted using bibliographic databases: Embase (1980-2016), OvidMEDLINE (1946 - 2016) and PsycINFO (1806 - 2016). The search terms, detailed in Table 1, were composed of three concepts: 'cognitive impairments', 'depression or anxiety' and 'psychosocial intervention'. Search strategies have been published for each database (Eady, Wilczynski, \& Haynes, 2008; Higgins \& Green, 2011; Wong, Wilczynski, \& Haynes, 2006) and are used in Cochrane reviews. These were used in the search to facilitate the identification of studies using a randomised design.

(2) Systematic reviews identified during the systematic search; and they were checked for studies that met the inclusion criteria.

(3) References in the identified studies were reviewed to find any further studies.

[Table 1 near here]

\section{Quality Rating of Studies}




\section{Treating anxiety and depression in dementia}

Cochrane's Risk of Bias tool, used to rate the quality of studies, was completed by two independent reviewers (DN and KL) using Review Manager (Revman) Version 5.3. The studies were rated as low, unclear or high risk of bias in areas such as: selection bias, performance bias, outcome bias, detection bias, attrition bias, reporting bias and other sources of bias. Low risk of bias suggests that the biases were unlikely to have an impact on the study results. Unclear risk of bias infers that there was not adequate information provided to evaluate all potential biases; therefore, it could raise questions about the reliability of the study results. High risk of bias infers that biases may have reduced the confidence in the study's results (Higgins \& Green, 2011). The results are illustrated in the 'Risk of Bias' graph and 'Risk of Bias' summary. The risk of bias informed the interpretation of the metaanalyses.

\section{Heterogeneity}

A statistical test (chi squared) measured the heterogeneity between the studies' results (Deeks, Higgins, \& Altman, 2008), with a low p-value indicating heterogeneity. The chi square test has been found to be poor at detecting true heterogeneity, especially when the meta-analysis includes a small number studies or when the overall sample size is small. A p value of less than 0.10 was used to determine if the studies were heterogeneous. However, heterogeneity is expected in meta-analyses due to variations in methodologies and clinical samples (Higgins, Thompson, Deeks, \& Altman, 2003). Therefore, the heterogeneity was quantified

using an $1^{2}$ statistic. Approximate guidelines for interpreting heterogeneity suggest that heterogeneity around $25 \%$ is low, medium heterogeneity is around $50 \%$, 
Treating anxiety and depression in dementia

substantial heterogeneity is around 75\% (Higgins, Thompson, Deeks, \& Altman, 2003).

\section{Data Extraction and Synthesis}

The population characteristics and the outcomes of each RCT were gathered by two independent reviewers (DN and KL). Discrepancies were resolved through discussion between reviewers. Revman was used to complete the meta-analyses. A random-effects model was used to present the overall estimated effect on depression and anxiety. As the studies under investigation used a range of scales, the analyses used the standardised mean difference scores, as opposed to the mean difference.

\section{Results}

After duplicates were removed, 2332 articles were extracted. Sixty-five were identified as relevant to the search criteria when the abstracts were read. The fulllength articles were checked against the inclusion and exclusion criteria. This resulted in seven articles selected for narrative review and six of these were included in the meta-analyses (See Figure 1).

[Figure 1 near here]

\section{Description of Studies}

The characteristics of the studies are detailed in Table 2. Participants were recruited from nursing homes, research centres and outpatient services. Eight studies recruited participants meeting criteria for dementia; with six of these studies specifying mild to moderate dementia in their inclusion criteria. No study met criteria for $\mathrm{MCl}$. Participants ranged from 73 to 88 years with a mean age of 82 . 
Interventions tested in the studies included cognitive behavioural therapy (Spector et al., 2015; Stanley et al., 2013), pleasant events behaviour therapy and problem solving behaviour therapy (Teri, Logsdon, Uomoto, \& McCurry, 1997a), music therapy (Guétin et al., 2009), Tai Chi (Cheng, Chow, Yu, \& Chan, 2012), exercise (Williams \& Tappen, 2008), problem adaptation therapy (PATH) (Kiosses et al., 2015) and a multicomponent intervention which incorporated CBT techniques, reminiscence, question asking reading, environmental supports and behavioural activity programs (Bailey, Stevens, LaRocca, \& Scogin, 2016). The length of intervention ranged from 12 to 16 sessions. Four studies used a wait list control or TAU and four used an active treatment condition.

[Table 2 near here]

\section{Quality Assessment and Risk of Bias}

The Cochrane's Risk of Bias tool was used to rate the quality of each study. Risk of Bias Items

Overall, $38 \%(n=3)$ of the studies did not report sufficient detail to determine the bias for both random sequence generation and allocation concealment. All the studies $(n=8)$ were unable to blind participants from the treatment conditions, due to the nature of the interventions. Most of the studies $(88 \%, n=7)$ provided details that the assessors, completing baseline and follow-up assessments, were blinded to treatment allocation; and were deemed to have a low risk of detection bias. All the studies were considered to have a low risk of bias for outcome data being incomplete and $63 \%(n=5)$ reported intention-to-treat (ITT) data. Most of the studies $(75 \%, n=6)$ were deemed to have a low risk of selective reporting because they reported all pre-specified outcomes; whilst two studies did not report the results of 


\section{Treating anxiety and depression in dementia}

one or more pre-specified outcomes. There were no other biases identified for the seven studies.

\section{Risk of Bias Summary}

Three studies were judged as having a low risk of bias in all domains apart from the blinding of participants (Guétin et al., 2009; Kiosses et al., 2015; Spector et al., 2015). Two studies were classified as unclear risk of bias, due to limitations such as ambiguities about allocation concealment (Stanley et al., 2013) and random sequence generation (Cheng et al., 2012). Three studies were classified as high risk of bias, due to selective reporting (Teri et al., 1997a; Williams \& Tappen, 2008) and not blinding the outcome assessment (Bailey et al., 2016).

\section{Systematic review and Meta-Analyses}

Interventions for Depression

End of treatment. Four studies with 159 participants that reported data on depression outcomes for psychosocial interventions were included in the metaanalysis, as compared to control interventions, within one week of completing treatment. One study provided inadequate information and could not be included in the meta-analysis(Teri, Logsdon, Uomoto, \& McCurry, 1997b). There were similarities in the intervention arms for the one study that used a three-arm design (Williams \& Tappen, 2008); therefore, they were pooled together for the metaanalyses. In another three-armed study (Cheng et al., 2012), one of the intervention arms (Mahjong) did not meet the outlined criteria for a psychosocial intervention. The intervention was not facilitated by a professional and was excluded from the metaanalysis. There was a significant $(p<.001)$ medium to large effect for psychosocial interventions in reducing depression, -0.62 ; (95\% confidence interval $(\mathrm{Cl}),-0.94$ to 0.29). There was no heterogeneity between studies $\left(1^{2}=0 \%\right)$, which suggests that 


\section{Treating anxiety and depression in dementia}

the effect is accurate and can be trusted (see Figure 2). Teri and colleagues' (1997) study, not included in the meta-analysis, found a significant reduction in depression scores in the treatment groups, as compared to the control groups $(p<0.001)$.

Bailey and colleagues' (2016) had a large between group effect size favoring a multicomponent intervention, as compared to treatment as usual. Three studies (Cheng et al., 2012; Kiosses et al., 2015; Williams \& Tappen, 2008) reported that the treatment (exercise/walking, Tai Chi, PATH) was neither superior nor inferior to the control group.

[Figure 2 near here]

Six-month follow up. Six-month follow up depression data was gathered for two of these studies (Cheng et al., 2012; Teri et al., 1997a). Cheng and colleagues (2012) reported that Tai Chi was neither superior not inferior to the control intervention. Teri and colleagues (1997), the study not included in the meta-analysis, found that the significant effect was maintained $(p<0.001)$.

\section{Interventions on Anxiety}

End of treatment. Three studies with 97 participants reported data on anxiety outcomes for psychosocial interventions, as compared to control interventions, within one week of completing treatment. These were all individual interventions (Guétin et al., 2009; Spector et al., 2015; Stanley et al., 2013). There was large significant $(p<.005)$ effect, -1.33 ; (95\% confidence interval $\mathrm{Cl},-2.21$ to -0.44$)$, for psychosocial interventions for reducing anxiety. There was a high amount of heterogeneity between studies $\left(1^{2}=72 \%\right.$ ) (see Figure 3 ), which suggests that the effect may not be accurate and should be interpreted with caution. 


\section{Treating anxiety and depression in dementia}

Two studies had a large between groups effect size favoring psychosocial interventions (CBT and music therapy), as compared to a control intervention (Guétin et al., 2009; Stanley et al., 2013). One study reported a medium to large between group effect size favoring a psychosocial intervention (CBT), as compared to a control intervention (Spector et al., 2015).

[Figure 3 near here]

Six-month follow up. Two of these studies reported data on anxiety outcomes at three and six month follow up (Guétin et al., 2009; Stanley et al., 2013). There was a significant large effect $(p<.05)$ for psychosocial interventions reducing anxiety $(-1.06$ $(95 \% \mathrm{Cl},-1.32$ to -0.67$))$. There was a medium to high amount of heterogeneity between studies $\left(I^{2}=64 \%\right)$, which suggests that the effect should be interpreted with caution (see Figure 4).

[Figure 4 near here]

\section{Discussion}

\section{Summary of Results}

This review evaluated the effectiveness of psychosocial interventions for depression and anxiety in PWD or MCl. Seven RCTs (five targeting depression and three anxiety) were selected for narrative review and six of these studies (four for depression, three for anxiety) were included in the meta-analyses.

\section{Depression}

A meta-analysis, from three RCTs, suggests that certain psychosocial interventions (Tai Chi, an exercise/walking intervention, PATH and a multicomponent intervention) are effective at improving depression symptoms in PWD, over the course of treatment. However, only one of these studies collected follow-up data (Cheng et al., 


\section{Treating anxiety and depression in dementia}

2012) and the treatment gains were not maintained (Cheng et al., 2012). Three (75\%) studies included in these meta-analyses for depression only recruited participants from nursing homes. One study (Teri et al., 1997a), not included in the meta-analysis, recruited participants from a community sample. It found that pleasant events behaviour therapy and problem solving behaviour therapy were effective at improving depression symptoms at the end of treatment; and this effect was maintained at follow up.

\section{Anxiety}

The meta-analysis from three RCTs, suggests that psychosocial interventions (music therapy and CBT) are effective at reducing anxiety symptoms in PWD at the end of treatment (Guétin et al., 2009; Spector et al., 2015; Stanley et al., 2013). These three studies were also individually effective at improving anxiety symptoms at the end of treatment. The meta-analysis, from two RCTs (music therapy and CBT), showed that treatment gains were maintained at follow-up (Guétin et al., 2009; Stanley et al., 2013). More specifically, music therapy maintained the effect (Guétin et al., 2009) and CBT did not (Stanley et al., 2013).

\section{Strengths and Limitations of the Included Studies}

A serious limitation of this meta-analysis was that the included studies had small sample sizes, which may have resulted in more extreme treatment effects. Therefore, future research needs to aim to recruit larger sample sizes. There was also variation in the included studies, in terms of the quality, population studied and the nature, intensity and frequency of the psychosocial intervention, which limited the conclusions made about specific type of interventions for specific populations. There was limited heterogeneity detected between study effects for the meta-analyses for 


\section{Treating anxiety and depression in dementia}

depression. However, there was a large amount of heterogeneity between study effects for the meta-analyses for anxiety, which suggests that the effect should be interpreted with caution.

The quality of the studies' methodology and reporting is consistent with the findings of previous reviews (Orgeta et al., 2014), which suggested that the quality of evidence is mixed, as a large proportion of the included studies were rated as being unclear or low quality. The quality of the studies for depression interventions was rated as poor, with three studies having a high risk of bias, one having an unclear risk of bias and one having a low risk of bias. The quality of studies, for anxiety interventions, was rated as good. The risk of bias was rated as low for two studies and unclear for one study and all studies used intention to treat analysis. Future RCTs are recommended to use a rigorous methodology and report the relevant information, as detailed in the Consolidated Standards of Reporting Trials (CONSORT).

Three studies did not detail information about power calculations therefore it was unclear if the sample size was sufficient to detect an effect (Bailey et al., 2016; Teri et al., 1997a; Williams \& Tappen, 2008). A large proportion of the studies $(57 \%, n=4)$ used a "treatment as usual" or "waitlist" control group, as compared to an active control group. It would be beneficial for future research to use an active control group to control for the placebo effect (Boot, Simons, Stothart, \& Stutts, 2013). The included studies were also limited to a single blind design due to the awareness of the group condition. 
Treating anxiety and depression in dementia

\section{Implications for Future Research}

\section{Depression}

Similar to other reviews (Orgeta et al., 2014; Regan \& Varanelli, 2013; Teri et al., 2005), there is some evidence to suggest that psychosocial interventions can reduce symptoms of depression in PWD who are depressed. These findings cannot be generalised to all PWD because most of the studies in the meta-analysis for depression recruited participants from nursing homes. Therefore, research is required on interventions for depression for PWD in community samples.

Most of the studies did not measure depression symptoms for long term follow up and the one study that did, found that the improvements were not maintained. Therefore, future research should aim to collect longitudinal data to inform the longer-term benefits of interventions for PWD. The only study that continued to show beneficial effect for a considerable amount of time after the end of the intervention, was the pleasant events behaviour therapy and problem solving behaviour therapy (Teri et al., 1997a), which was not included in the meta-analysis. This study recruited participants from a community sample and their mean age was younger than the studies included in the meta-analysis; potentially suggesting that the benefits of interventions on depression symptoms may be better maintained in younger PWD in community samples, a potential focus for future research.

\section{Anxiety}

All the included studies had a medium-to-large (Spector et al., 2015) or large effect size (Guétin et al., 2009; Stanley et al., 2013). Therefore, music therapy and CBT interventions for PWD with anxiety should be prioritised for research. The evidence for music therapy is perhaps particularly strong as it used an active control group 


\section{Treating anxiety and depression in dementia}

(reading group) and it was also found to maintain its large effect at six month follow up (Guétin et al., 2009). This may be the result of participants being offered more sessions (16 sessions), as compared to CBT (10-12 sessions) (Spector et al., 2015; Stanley et al., 2013). Therefore, it may be beneficial for future research to investigate if interventions that provide more sessions to PWD are more effective than those that provide fewer sessions.

\section{Implications for Practice}

\section{Depression}

The multicomponent intervention (Bailey et al., 2016) had the largest effect size for depression interventions, despite using intention to treat analysis. However, the study was rated as having a high risk of bias. This intervention incorporated CBT techniques, reminiscence, environmental supports and a behavioural activity program. This intervention may be beneficial for PWD residing in nursing homes. Future research should identify which component of this intervention was the agent for change.

Approximately half of the interventions (multicomponent intervention, Tai Chi) had low attrition rates (Bailey et al., 2016; Cheng et al., 2012), which is positive for practice. However, attrition rates in RCTs may not be comparable to attrition rates in normal practice therefore it is recommended that the implementation of these interventions are tested in services.

Anxiety

Music therapy (Guétin et al., 2009) was found to improve anxiety for PWD residing in nursing homes, with a large effect size. Whereas CBT was found to improve anxiety 


\section{Treating anxiety and depression in dementia}

for PWD from a community setting, with a medium-to-large (Spector et al., 2015) and large (Stanley et al., 2013) effect size. Music therapy and CBT interventions for PWD with anxiety should be prioritised for practice.

\section{Completeness of Evidence}

Most of the included studies recruited participants with mild to moderate dementia. The review did not identify any RCTs that were aimed at people with $\mathrm{MCI}$ or people with late stage dementia. There were four potential studies that were identified; although they recruited participants with cognitive impairment which did not meet the established criteria for MCI (Alexopoulos et al., 2011; Areán et al., 2010; Kiosses et al., 2015; Kiosses, Arean, Teri, \& Alexopoulos, 2010). As depression and anxiety have been found to increase the risk of dementia in people with $\mathrm{MCl}$ (Cooper et al., 2015), it is important that psychosocial interventions for depression and anxiety are available for this population.

Seven studies, that tested psychosocial intervention for PWD, were identified. The depression outcomes for three studies and the anxiety outcomes for three studies were pooled together. However data from one study failed to be obtained despite contacting the author (Teri et al., 1997a). Therefore, the study could only be included in the narrative review.

\section{Conclusion}

This systematic review with meta-analyses was distinctive because it aimed to compare the efficacy of psychosocial interventions on symptoms of depression and anxiety in PWD or $\mathrm{MCl}$ who were depressed and/or anxious. Preliminary evidence suggests that certain psychosocial interventions are effective at improving 


\section{Treating anxiety and depression in dementia}

depression and anxiety symptoms in PWD over the course of treatment. The quality of evidence was good for the interventions targeting anxiety, whereas it was poor for the interventions targeting depression. It was recommended that music therapy and CBT interventions for PWD and anxiety should be prioritised for research and practice.

\section{Acknowledgements}

Conflict of Interest: The authors declare no conflict of interest.

Author Contributions: All authors have had a role in the planning, execution, or analysis of the study and have approved the submitted version of the manuscript. The specific contribution of each author was: study design: DN, AS and JS; interpretation of data: $\mathrm{DN}$ and $\mathrm{KL}$; drafting of the first version of the manuscript: $\mathrm{DN}$; critical revision of the manuscript: all authors; statistical analysis: DN; study supervision: AS, JS and EA.

Sponsor's Role: There was no sponsor for this research.

\section{References}

Alexopoulos, G. S., Raue, P. J., Kiosses, D. N., Mackin, R. S., Kanellopoulos, D., McCulloch, C., \& Areán, P. A. (2011). Problem-solving therapy and supportive therapy in older adults with major depression and executive dysfunction: effect on disability. Archives of General Psychiatry, 68(1), 33-41.

Areán, P. A., Raue, P., Mackin, R. S., Kanellopoulos, D., McCulloch, C., \& Alexopoulos, G. S. (2010). Problem-solving therapy and supportive therapy in older adults with major depression and executive dysfunction. American Journal of Psychiatry, 167(11), 1391-1398.

Bailey, E. M., Stevens, A. B., LaRocca, M. A., \& Scogin, F. (2016). A Randomized Controlled Trial of a Therapeutic Intervention for Nursing Home Residents With 


\section{Treating anxiety and depression in dementia}

Dementia and Depressive Symptoms. Journal of Applied Gerontology, 1-14.

Boot, W. R., Simons, D. J., Stothart, C., \& Stutts, C. (2013). The Pervasive Problem

With Placebos in Psychology. Perspectives on Psychological Science, 8(4), 445-454.

Cheng, S. T., Chow, P. K., Yu, E. C. S., \& Chan, A. C. M. (2012). Leisure Activities Alleviate Depressive Symptoms in Nursing Home Residents With Very Mild or Mild Dementia. The American Journal of Geriatric Psychiatry, 20(10), 904-908.

Cooper, C., Sommerlad, A., Lyketsos, C. G., \& Livingston, G. (2015). Modifiable predictors of dementia in mild cognitive impairment: A systematic review and meta-analysis. American Journal of Psychiatry, 172(4), 323-334.

Deeks, J. J., Higgins, J. P., \& Altman, D. G. (2008). Analysing Data and Undertaking Meta-Analyses. In Cochrane Handbook for Systematic Reviews of Interventions: Cochrane Book Series (pp. 243-296). Chichester, UK: John Wiley \& Sons, Ltd.

Devanand, D. P., Pelton, G. H., Marston, K., Camacho, Y., Roose, S. P., Stern, Y., \& Sackeim, H. A. (2003). Sertraline treatment of elderly patients with depression and cognitive impairment. International Journal of Geriatric Psychiatry, 18(2), $123-130$.

Eady, A. M., Wilczynski, N. L., \& Haynes, R. B. (2008). PsyclNFO search strategies identified methodologically sound therapy studies and review articles for use by clinicians and researchers. Journal of Clinical Epidemiology, 61(1), 34-40.

Forsman, A. K., Schierenbeck, I., \& Wahlbeck, K. (2011). Psychosocial interventions for the prevention of depression in older adults: Systematic review and metaanalysis. Journal of Aging and Health, 23(3), 387-416.

Frederiksen, Y., Farver-Vestergaard, I., Skovgard, N. G., Ingerslev, H. J., \& Zachariae, R. (2015). Efficacy of psychosocial interventions for psychological 


\section{Treating anxiety and depression in dementia}

and pregnancy outcomes in infertile women and men: a systematic review and meta-analysis. British Medical Journal Open, 5(1), e006592-e006592.

Guétin, S., Portet, F., Picot, M. C., Pommié, C., Messaoudi, M., Djabelkir, L., ... Touchon, J. (2009). Effect of music therapy on anxiety and depression in patients with Alzheimer's type dementia: randomised, controlled study. Dementia and Geriatric Cognitive Disorders, 28(1), 36-46.

Higgins, J., \& Green, S. (2011). Cochrane handbook for systematic reviews of interventions.

Higgins, J., Thompson, S., Deeks, J., \& Altman, D. (2003). Measuring inconsistency in meta-analyses. British Medical Journal, 327(7414), 557-560.

Kales, H. C., Chen, P., Blow, F. C., Welsh, D. E., \& Mellow, A. M. (2005). Rates of Clinical Depression Diagnosis, Functional Impairment, and Nursing Home Placement in Coexisting Dementia and Depression. The American Journal of Geriatric Psychiatry, 13(6), 441-449.

Kane, M., \& Terry, G. (2015). Social Care Online | Dementia 2015: aiming higher to transform lives.

Kaplan, D. B., \& Berkman, B. (2011). Dementia care: A global concern and social work challenge. International Social Work, 54(3), 361-373.

Kiosses, D. N., Arean, P. A., Teri, L., \& Alexopoulos, G. S. (2010). Home-delivered problem adaptation therapy (PATH) for depressed, cognitively impaired, disabled elders: A preliminary study. The American Journal of Geriatric Psychiatry: Official Journal of the American Association for Geriatric Psychiatry, 18(11), 988-98.

Kiosses, D. N., Rosenberg, P. B., McGovern, A., Fonzetti, P., Zaydens, H., \& Alexopoulos, G. S. (2015). Depression and Suicidal Ideation During Two 
Treating anxiety and depression in dementia

Psychosocial Treatments in Older Adults with Major Depression and Dementia. Journal of Alzheimer's Disease, 48(2), 453-462. http://doi.org/10.3233/JAD150200

Kiosses, D., Ravdin, L. D., Gross, J. J., Raue, P., Kotbi, N., \& Alexopoulos, G. S. (2015). Problem adaptation therapy for older adults with major depression and cognitive impairment: A randomized clinical trial. JAMA Psychiatry, 72(1), 2230.

Leong, C. (2014). Antidepressants for Depression in Patients with Dementia: A Review of the Literature. The Consultant Pharmacist, 29(4), 254-263.

Lyketsos, C. G., Lopez, O., Jones, B., Fitzpatrick, A. L., Breitner, J., \& DeKosky, S. (2002). Prevalence of Neuropsychiatric Symptoms in Dementia and Mild Cognitive Impairment. JAMA, 288(12), 1475.

McCurry, S. M., Gibbons, L. E., Logsdon, R. G., \& Teri, L. (2004). Anxiety and nighttime behavioral disturbances. Awakenings in patients with Alzheimer's disease. Journal of Gerontological Nursing, 30(1), 12-20.

Monastero, R., Mangialasche, F., Camarda, C., Ercolani, S., \& Camarda, R. (2009). A Systematic Review of Neuropsychiatric Symptoms in Mild Cognitive Impairment. Journal of Alzheimer's Disease, 18, 11-30.

Moretti, R., Torre, P., Antonello, R. M., \& Pizzolato, G. (2006). Atypical neuroleptics as a treatment of agitation and anxiety in Alzheimer's disease: risks or benefits. Expert Review of Neurotherapeutics, 6(5), 705-710.

Olazarán, J., Reisberg, B., Clare, L., Cruz, I., Peña-Casanova, J., del Ser, T., ... Muñiz, R. (2010). Nonpharmacological Therapies in Alzheimer's Disease: A Systematic Review of Efficacy. Dementia and Geriatric Cognitive Disorders, $30(2), 161-178$. 


\section{Treating anxiety and depression in dementia}

Orgeta, V., Qazi, A., Spector, A. E., \& Orrell, M. (2014). Psychological treatments for depression and anxiety in dementia and mild cognitive impairment. The Cochrane Database of Systematic Reviews, (1), 1-3.

Petersen, R. C., Caracciolo, B., Brayne, C., Gauthier, S., Jelic, V., \& Fratiglioni, L. (2014). Mild cognitive impairment: a concept in evolution. Journal of Internal Medicine, 275(3), 214-228. http://doi.org/10.1111/joim.12190

Petticrew, M., \& Roberts, H. (2006). Systematic reviews in the social sciences : a practical guide. Oxford: Blackwell.

Rapp, M. A., Schnaider-Beeri, M., Wysocki, M., Guerrero-Berroa, E., Grossman, H. T., Heinz, A., \& Haroutunian, V. (2011). Cognitive Decline in Patients With Dementia as a Function of Depression. The American Journal of Geriatric Psychiatry, 19(4), 357-363.

Regan, B., \& Varanelli, L. (2013). Adjustment, depression, and anxiety in mild cognitive impairment and early dementia: a systematic review of psychological intervention studies. International Psychogeriatrics, 25(15), 1963-84.

Sepehry, A. A., Lee, P. E., Hsiung, G. Y. R., Beattie, B. L., \& Jacova, C. (2012). Effect of Selective Serotonin Reuptake Inhibitors in Alzheimer's Disease with Comorbid Depression. Drugs \& Aging, 29(10), 793-806.

Shin, I.-S., Carter, M., Masterman, D., Fairbanks, L., \& Cummings, J. L. (2005). Neuropsychiatric Symptoms and Quality of Life in Alzheimer Disease. The American Journal of Geriatric Psychiatry, 13(6), 469-474.

Spector, A., Charlesworth, G., King, M., Lattimer, M., Sadek, S., Marston, L., ... Orrell, M. (2015). Cognitive-behavioural therapy for anxiety in dementia: pilot randomised controlled trial. The British Journal of Psychiatry: The Journal of Mental Science, 206(6), 509-16. 
Stanley, M. A., Calleo, J., Bush, A. L., Wilson, N., Snow, A. L., Kraus-Schuman, C., ... Kunik, M. E. (2013). The Peaceful Mind Program: A Pilot Test of a CognitiveBehavioral Therapy-Based Intervention for Anxious Patients with Dementia. The American Journal of Geriatric Psychiatry, 21(7), 696-708.

Starkstein, S. E., Jorge, R., Petracca, G., \& Robinson, R. G. (2007). The Construct of Generalized Anxiety Disorder in Alzheimer Disease. The American Journal of Geriatric Psychiatry, 15(1), 42-49.

Teri, L., Logsdon, R. G., Uomoto, J., \& McCurry, S. M. (1997a). Behavioral Treatment of Depression in Dementia Patients: A Controlled Clinical Trial. The Journals of Gerontology Series B: Psychological Sciences and Social Sciences, 52(4), 159-166.

Teri, L., Logsdon, R., Uomoto, J., \& McCurry, S. (1997b). Behavioral treatment of depression in dementia patients: A controlled clinical trial. Journals of Gerontology - Series B Psychological Sciences and Social Sciences, 52(4), 159-166.

Teri, L., McKenzie, G., \& LaFazia, D. (2005). Psychosocial treatment of depression in older adults with dementia. Clinical Psychology: Science and Practice, pp. 303-316.

UN Department of Economic and Social Affairs. (2013). World Population Ageing. Williams, C. L., \& Tappen, R. M. (2008). Exercise training for depressed older adults with Alzheimer's disease. Aging \& Mental Health, 12(1), 72-80.

Wong, S. S.-L., Wilczynski, N. L., \& Haynes, R. B. (2006). Developing optimal search strategies for detecting clinically sound treatment studies in EMBASE. Journal of the Medical Library Association, 94(1), 41-7. 